\title{
Correction to: Interplay between low plasma RANKL and VDR-FokI polymorphism in lumbar disc herniation independently from age, body mass, and environmental factors: a case-control study in the Italian population
}

\author{
Veronica Sansoni $^{1}$. Silvia Perego ${ }^{1}$ - Alessandra Colombini ${ }^{1}$. Giuseppe Banfi ${ }^{1,2} \cdot$ Marco Brayda-Bruno $^{3}$. \\ Giovanni Lombardi ${ }^{1}$ (D)
}

Published online: 7 February 2020

(c) Springer-Verlag GmbH Germany, part of Springer Nature 2020

\section{Correction to: Eur Spine J (2016) 25:192-199}

https://doi.org/10.1007/s00586-015-4176-7

Under the headline "Correlation of RANKL concentrations and VDR-FokI polymorphism on disc herniation" in the description text for Table 2, the term "allelic frequency" was used erroneously for "genotypic frequency".
The complete corrected Table 2 is given below.

Table 2 Genotype frequencies within the study population

\begin{tabular}{lllr}
\hline & \multicolumn{3}{l}{$V D R$-FokI genotypes } \\
\cline { 2 - 4 } & $F F$ & $F f$ & \multicolumn{1}{c}{$f f$} \\
\hline All $(n=220)$ & 43.64 & 43.18 & 13.18 \\
M $(n=110)$ & 23.64 & 20.45 & 5.91 \\
F $(n=110)$ & 20.00 & 22.73 & 7.27 \\
Cases $(n=110)$ & 47.27 & 40.00 & 12.73 \\
M $(n=55)$ & 26.36 & 20.91 & 2.73 \\
F $(n=55)$ & 20.91 & 19.09 & 10.00 \\
Controls $(n=110)$ & 40.00 & 46.36 & 13.64 \\
M $(n=55)$ & 20.91 & 20.00 & 9.09 \\
F $(n=55)$ & 19.09 & 26.36 & 4.55 \\
\hline
\end{tabular}

The frequency ( $\%$, relative to the size of the subcohort) of the different genotypes associated with the FokI polymorphisms of the VDR gene in the different cohorts

$M$ males, $F$ females

Publisher's Note Springer Nature remains neutral with regard to jurisdictional claims in published maps and institutional affiliations.
The original article can be found online at https://doi.org/10.1007/ s00586-015-4176-7.

Giovanni Lombardi

giovanni.lombardi@grupposandonato.it

Laboratory of Experimental Biochemistry and Molecular Biology, I.R.C.C.S. Istituto Ortopedico Galeazzi, Milan, Italy

2 Vita-Salute San Raffaele University, Milan, Italy

3 Scoliosis Unit, Department of Orthopedics and Traumatology-Spine Surgery III, I.R.C.C.S. Istituto Ortopedico Galeazzi, Milan, Italy 